Article

\title{
Optical 3-D Profilometry for Measuring Semiconductor Wafer Surfaces with Extremely Variant Reflectivities
}

\author{
Liang-Chia Chen ${ }^{1,2, * \mathbb{C}}$, Duc-Hieu Duong ${ }^{2}$ and Chin-Sheng Chen ${ }^{2}$ (I) \\ 1 Department of Mechanical Engineering, National Taiwan University, No. 1, Section 4, Roosevelt Rd, Da'an \\ District, Taipei City 10617, Taiwan \\ 2 Graduate Institute of Automation Technology, College of Mechanical and Electrical Engineering, National \\ Taipei University of Technology, No. 1, Section 3, Zhong-Xiao E. Rd, Da'an District, Taipei City 10608, \\ Taiwan; duongduchieu85@gmail.com (D.-H.D.); saint@mail.ntut.edu.tw (C.-S.C.) \\ * Correspondence: lchen@ntu.edu.tw; Tel.: +886-2-23620032
}

Received: 2 April 2019; Accepted: 14 May 2019; Published: 19 May 2019

\begin{abstract}
A new surface profilometry technique is proposed for profiling a wafer surface with both diffuse and specular reflective properties. Most moiré projection scanning techniques using triangulation principle work effectively on diffuse reflective surfaces, on which the reflected light beams are assumed to be well captured by optical sensors. In reality, this assumption is no longer valid when measuring a semiconductor wafer surface having both diffuse and specular reflectivities. To resolve the above problem, the proposed technique uses a dual optical sensing configuration by engaging two optical sensors at two different viewing angles, with one acquiring diffuse reflective light and the other detecting at the same time specular surface light for achieving simultaneous full-field surface profilometry. The deformed fringes measured by both sensors could be further transformed into a 3-D profile and merged seamlessly for full-field surface reconstruction. Several calibration targets and industrial parts were measured to evaluate the feasibility and accuracy of the developed technique. Experimental results showed that the technique can effectively detect diffuse and specular light with repeatability of one standard deviation below $0.3 \mu \mathrm{m}$ on a specular surface and $2.0 \mu \mathrm{m}$ on a diffuse wafer surface when the vertical measuring range reaches $1.0 \mathrm{~mm}$. The present findings indicate that the proposed technique is effective for 3-D microscale surface profilometry in in-situ semiconductor automated optical inspection (AOI).
\end{abstract}

Keywords: semiconductor; surface profilometry; moiré projection; 3-D measurement; automated optical inspection (AOI)

\section{Introduction}

Measuring the 3-D profile of an object surface has become increasingly important in industrial automation, particularly in in-situ product inspection. 3-D surface measurement could be generally classified into two major categories: tactile and non-contact measuring conditions. Coordinate Measuring Machine (CMM) is a general system using both contact and non-contact techniques to measure 3-D profiles. Tactile sensing cannot accurately measure soft object or thin-film surfaces as the probe may scratch or deform the measured surface. In contrast, non-contact techniques can effectively measure surfaces with high spatial resolution and are useful for measuring soft or flexible objects. For non-contact measurement, optical triangulation techniques, such as stereo vision, laser scanning, and structured light techniques, are widely used in industries. However, optical techniques often require uniform surface light reflection to ensure reliable sensing. The absence of such condition would pose difficulty for using optical triangulation techniques to measure a surface with both diffuse and 
specular reflectivities. One of the significant challenges in optical surface measurement lies in dealing with reflectivity variations of the object surfaces to be tested, such as a semiconductor wafer surface being engraved or marked by laser etching. Measuring objects with high reflectivity variations, such as highly shiny or scattering surfaces, is a critical issue to ensuring measurement accuracy and product quality assurance under automated optical inspection (AOI). Poor 3-D profiling results are common when encountering high reflectance variances on the wafer surface to be tested or reconstructed for 3-D printing or other purposes.

One of the popular 3-D optical surface measurement techniques is structured light projection, which is widely employed in industrial testing applications. The techniques basically work according to the triangulation measurement principle with the assumption of uniform light reflectivity on the tested object for ensuring capture of the deformed fringe reflecting from the tested surface. However, in reality, such assumption is not applicable in face of high reflectivity variations on the surface. For example, most industrial IC chips are fabricated using a silicon wafer substrate with an extremely shiny surface and are also embedded with metal bumps having a scattering surface reflectance. Under such condition, the reflected lights from the tested surface are rather complex and emitting in different directions. Consequently, the detecting sensor cannot receive accurate phase information for reconstructing surface morphology.

Structured fringe projection is a full-field optical technique for measuring 3-D object surfaces. Owing to its advantages of fast speed, high accuracy, being non-destructive, and full-field testing, fringe projection technique (FPP) has become one of the most promising 3-D surface profiling techniques in 3-D AOI. For obtaining the object shape, the common 3-D measurement techniques include mainly phase shifting profilometry (PSP) [1] and Fourier transform profilometry (FTP) [2]. In general, these techniques project structured light patterns, also called fringes, onto the object surface. Owing to optical path difference (OPD) between inspecting beams, the fringe is deformed according to the 3-D shape of the object surface. To reconstruct the 3-D shape of the object surface, one phase map is computed using a wrapping algorithm, and object height can be extracted using the phase unwrapping algorithm [3] and phase-to-height transformation. PSP is widely employed in industrial inspection because of its promising accuracy and high spatial resolution [4]. It uses a time-resolved wrapping strategy to extract phase information for reconstruction of object profiles. Different from PSP, FTP records distorted fringe images and analyzes object height information using phase modulation and demodulation to acquire a phase map from distorted fringes. The unique advantage of FTP is its one-shot capability of 3-D surface imaging. However, for exacting 3-D profile reconstruction, one of the key issues in FTP is to extract accurately the first-order spectrum from the spectral domain. The extraction would require an adequate band-pass filter to obtain accurate spectral information from the first-order spectrum and separate it from the background spectrum (zero-order spectrum). Conditions for separating these spectrum regions have been investigated by considering the minimum distance between spectrum regions [5-7]. The single elliptic band-pass filter [8] and double orthogonal elliptic band-pass filter $[9,10]$ have been developed to extract accurately the first-order spectrum from the spectral domain. Nevertheless, FTP still lacks a robust algorithm for detecting the first-order spectrum to reconstruct accurately 3-D profiles. Apart from these methods, scanning white light interferometry (SWLI) is powerful for its phase unambiguity and nano-scale depth resolution [11]. It has been widely applied for precise sub-micro-scale and nano-scale surface profilometry, especially in semiconductor microstructure metrology. The method is mainly used for review or off-line metrology since its scanning efficiency is limited by vertical scanning required.

A major challenge for 3-D scanning techniques using FPP is the presence of specular reflectance on the tested object surface. Specular highlights occur on object surfaces when the specular element of reflection is dominant. Specular highlight can easily saturate the detected image and seriously deteriorate image quality. In the literature, several techniques have been proposed for resolving the problem when measuring specular targets. All these techniques can effectively reduce specular effects to different extents but cannot resolve the problem completely. One of the proposed techniques was 
to separate specular components from diffuse components and remove the specular components using polarizers [12-15]. Tan and Ikeuchi relied on image color properties when transferring image color to the hue domain [16]. This technique effectively eliminated image-saturated regions mainly because the saturation value of every pixel was made constant for achieving maximum chromaticity while keeping their hue. Other techniques, such as using multi-exposure time [17] or projecting special structured light with strip edges [18] have been experimented for handling non-uniform light reflection. Phase measuring deflectometry (PMD) is a specular surface measuring technique using a Thin-Film-Transistor Liquid-Crystal Display (TFT LCD) screen as the pattern-projecting light source $[19,20]$. The maximum detectable surface curvature is currently limited by the LCD light dispersion angle. More recently, single-shot deflectometry using a single composite pattern was developed for one-shot measurement of three-dimensional imaging using Fourier transform (FT) and spatial carrier-frequency phase-shifting (SCPS) techniques [21]. However, generally for PMD, due to demanding computation in slope integration, the absolute shape measurement may not be accurately achieved. Thus, apart from visible moiré fringe projection, Wiedenmann et al. used high-power infrared laser pattern to project on the measured object surface, in which the projected laser power was partly transferred into dispersive heat [22]. Since heat dispersion can be generated from a highly specular surface, extremely dark or transparent materials can be observed clearly by a proper heat sensor [23]. Phase shifting technique can be employed to measure 3-D profile according to distortion of the heat pattern on the object surface. The current limitation of the method is its low measuring accuracy, which still requires further improvement. Up to date, some light separation strategies using light property analysis were developed, including separation specular reflection using color analysis [3], examining chromaticity and intensity value of specular and diffuse pixels [16], employing polarization to separate reflection components [24] or using environmental structured illumination [25] techniques. Although these methods have been effective to some extents in separating diffuse from specular light, they basically assume either uniform surface reflectance or specific surface light reflectance conditions, which may not be applicable for many in-situ testing applications.

Many semiconductor parts with variant reflectivities need to be inspected in in-situ manufacturing processes for strict dimensional compliance. These parts often comprise a body (such as epoxy and silicon), metal bumps, and pads (such as lead and solder), each of which may have complicated geometric shapes with different orientations with respect to the detecting optical sensor [26]. Moreover, the most challenging task is to overcome extremely variant wafer surface reflectivities, ranging widely from diffuse to specular conditions. In 3-D inspection, the 3-D surface profilometric technique of the tested objects often projects a structured light pattern with a desirable assumption that a non-absorbent and perfect diffusely scattered model of the surface under test (SUT) is observed. However, in reality, this assumption may not be true for many actual cases. In optical triangulation detection, viewing the measured surface from a sensing angle can pose a huge measuring uncertainty in ensuring detection of reflecting light due to variant surface reflectance properties. The extent of reflecting light detected by imaging sensors can significantly affect the success of surface measurement. Most existing techniques discussed above can resolve the light specular problem only for a surface with uniform reflectivity. For objects with non-uniform surface reflectivity, the captured image contrast becomes poor or even non-detectable.

Therefore, it is of need to develop an effective technique that can overcome the complex reflectivity problem encountered in optical surface profilometry, especially for in-situ semiconductor wafer surface inspection. The article proposes a dual sensing strategy to overcome the 3-D surface profilometric problem encountered in semiconductor wafer surface. The rest of this paper is organized as follows. Section 2 presents the proposed method for 3-D profilometry using dual sensing. The experimental design and measured results are shown and analyzed in Section 3. Finally, the conclusions are summarized in Section 4. 


\section{Developed 3-D Profilometry Using Dual Sensing}

In structured fringe projection, the angle between the projection and the sensing detection plays an important role in determining the reflecting direction. Figure 1a shows the fringe image captured from a semiconductor wafer surface detected using a laser-etched microscale grove with the viewing angle set at zero. As can be seen, the groove area is rather clear but the remaining wafer specular area is almost non-detectable. Therefore, the groove area can be seen with an adequate exposure time because diffuse light is generally weak. With larger viewing angle, the wafer surface becomes clearer with a better image contrast (see Figure $1 b, c$ ). Illustration shown from Figure $1 b-d$ represents a transition in imaging with increase in viewing angle. When the reflectance relation follows the light reflectance rule, the fringe contrast on the wafer surface reaches its maximum as illustrated in Figure 1e. Figure $1 \mathrm{f}$ represents the case when the sensor becomes over saturated owing to excessive exposure time. Figure 1 illustrates the critical reflectance condition of the tested surface under inspection. Figure 2 shows the general reflection model of diffuse and specular components when the light is incident from an angle.

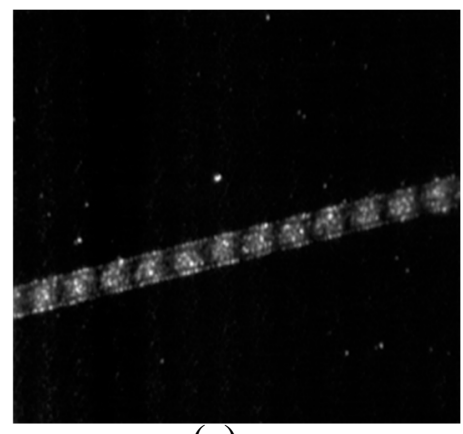

(a)

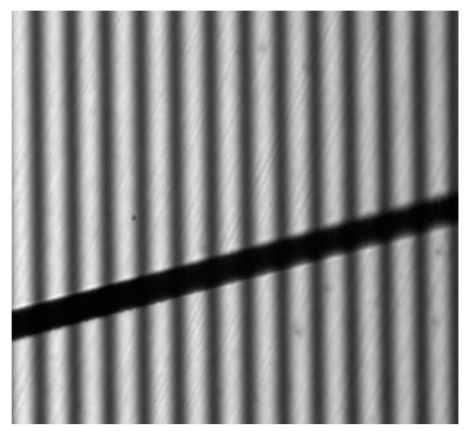

(d)

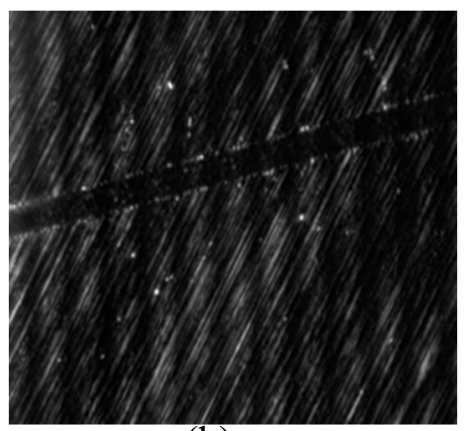

(b)

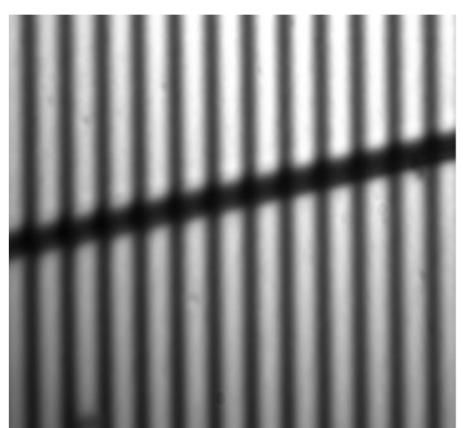

(e)

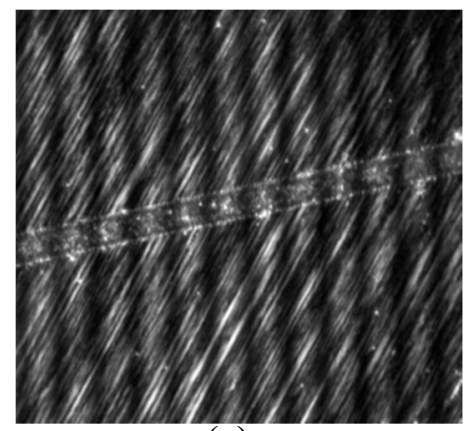

(c)

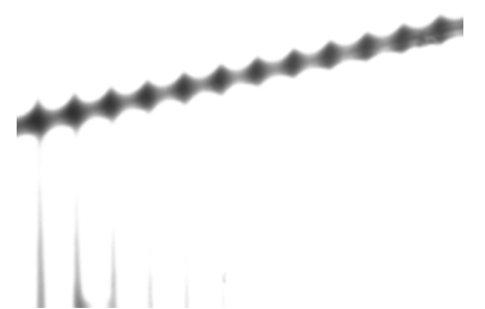

(f)

Figure 1. Images of wafer surface etched with a laser-etched groove and captured at different viewing angles: (a) viewing angle $\pm 1^{\circ}$, exposure time $420 \mathrm{~ms}$; (b) viewing angle $12 \pm 1^{\circ}$, exposure time $200 \mathrm{~ms}$; (c) viewing angle $25 \pm 1^{\circ}$, exposure time $80 \mathrm{~ms}$; (d) viewing angle $45 \pm 1^{\circ}$, exposure time $20 \mathrm{~ms}$; and (e) viewing angle $45 \pm 1^{\circ}$, exposure time $50 \mathrm{~ms}$.

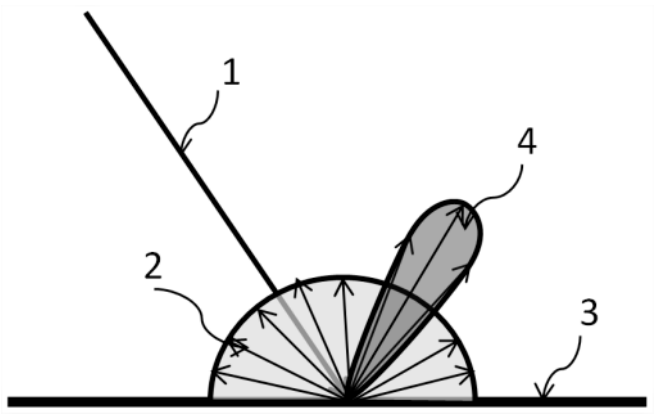

Figure 2. Reflection model of diffuse and specular components. 
To resolve the above issue, this article presents a new optical measuring configuration to capture both of specular and diffuse light reflecting from wafer surfaces. A dual sensing strategy is proposed to detect the light reflection from wafer surfaces when structured fringe projection is used to measure and reconstruct 3-D surfaces. The proposed system design and measuring principle are detailed in the following sessions.

\subsection{Optical System Setup}

Figure 3 illustrates the optical system configuration of the developed 3-D profilometry. As can be seen, it includes a low-coherent light source (1), a diffuser (2), which is collimated by a lens module (3), a sinusoidal grating (4), and a linear translator (5) for generating an accurate shifted phase. To achieve full-field surface profilometry using PSP, the light source is a low-coherent light source such as LED (light-emitting diode), instead of a coherent one like lasers. In the system, the structured light generated by the incident optical module passes through a telecentric lens (6) and is projected onto the measured surface (8) defined by the reference plane (7). The reflected light passes through two objectives (12 and 13) and is captured by two individual optical sensing cameras (10 and 11). The first objective (12) is arranged in vertical direction to capture the diffuse light while the second objective is set to a reflection angle with respect to the incident projection light angle, so that specular light can be well captured. With such an optical configuration, both diffuse and specular light beams reflected can be well detected by their corresponding objectives. When the projected sinusoidal fringe (9) hits the measured wafer surface, it becomes a deformed fringe according to the phase difference generated by the optical path difference (OPD) between the object profile and the reference plane. The deformed fringe image is transferred to a computer (14) and the phase map is then computed with multi-phase shifting and the wrapping principle using a set of deformed fringe images. Subsequently, phase unwrapping and phase-to-height transformation are performed to extract the profile of the measured surface.

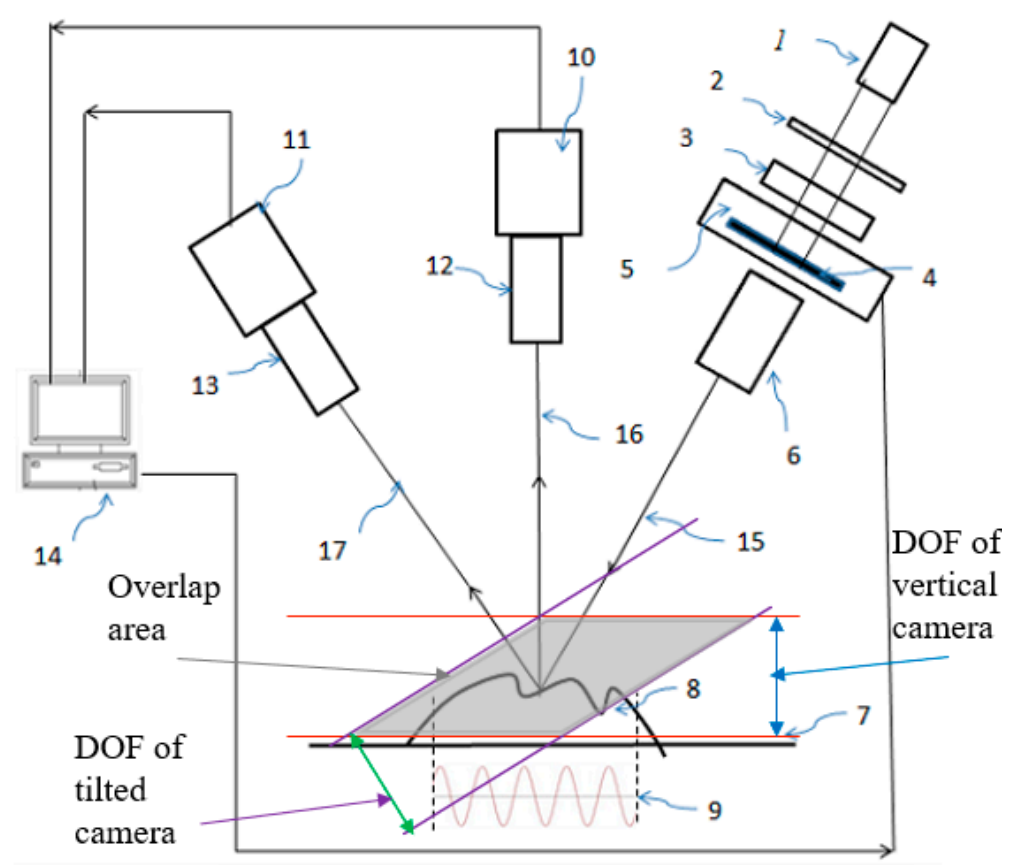

Figure 3. Configuration of the developed dual-sensing measurement system.

All the optical lenses employed in the developed system are telecentric to ensure the formation of a constant image size along the depth of field (DOF) of the measurement. When the measured surface located inside the overlapped with the DOF of the two cameras (indicated as the gray zone in Figure 3), the captured image size can be kept constant, so the FOV is kept as a rectangular or square shape according to the image sensor's design. Meanwhile, to keep the focus plane of the imaging 
sensor to locate at the same focus plane with the projecting light side, the image sensor can titled with the principle optical axis by an adequate angle to compensate the tilting effect. Thus, both of the focus planes (light projection and imaging) are kept at the same focus plane and the image shape are rectangular or square in the same FOV. Meanwhile, an adequate camera calibration can be performed to identify all the intrinsic and extrinsic parameters of the coordinate transform, so 3D point cloud can be transformed with image coordinates accurately.

\subsection{Measuring Principle}

\subsubsection{Fundamentals in PSP}

Using PSP technique for 3-D shape measurement involves several procedures, including projecting sinusoidal fringe patterns onto the object surface, taking the images of the reflected distorted pattern on the object surface, and then calculating the phase map from these captured images. Height information is extracted from the phase map by phase unwrapping and phase-to-height transformation. The calibration coefficients are then employed to transform the 3-D coordinates in image coordinates into world coordinates $(x, y, z)$ of each measured point. The phase $\phi(x, y)$ can be computed using light intensities of captured images set by phase-shifted fringe projection. The five-step phase-shifting technique is commonly used because of its accuracy and efficiency. The intensities of the five channels in each pattern can be modeled as follows:

$$
I_{i}(x, y)=I_{b}(x, y)+I_{m}(x, y) \cos \left[\phi(x, y)+\delta_{i}\right]_{(i=1 \sim 5)}
$$

where $\phi(x, y)$ is the phase value used by the phase-shifting algorithm in $(x, y)$ location, $\delta_{i}$ is the phase-shift, $I_{i}(x, y)$ denotes the intensities of the five captured images for each pixel position, $I_{m}(x, y)$ and $I_{b}(x, y)$ are the intensity modulation and the average intensity for each pixel, respectively.

The phase value of each pixel $\phi(x, y)$ can be resolved using Equation (1) as:

$$
\phi(x, y)=-\tan ^{-1}\left[\frac{\sum_{i=1}^{N} I_{i}(x, y) \sin \left(\delta_{i}\right)}{\sum_{i=1}^{N} I_{i}(x, y) \cos \left(\delta_{i}\right)}\right]
$$

where $\delta_{i}, i=0,1,2, \ldots N$, are the phase-shift values of each channel.

For the five-step phase-shifting technique $(N=5)$ and $\delta_{1}=0^{\circ}, \delta_{2}=90^{\circ}, \delta_{3}=180^{\circ}, \delta_{4}=$ $270^{\circ}, \delta_{5}=360^{\circ}$, the phase value $\phi(x, y)$ can be computed using the intensity of each channel and the corresponding phase-shift $\delta_{i}$ from the five-step phase-shifting technique shown in Equation (2).

With the phase value $\phi(x, y)$ being a function of arctangent, the phase value can be obtained in a range from $-\pi$ to $\pi$ for phase wrapping. Consequently, if the pixel-based phase difference is larger than $2 \pi$, the wrapping phase becomes discontinuous and ambiguous. To obtain a continuous phase map, a phase unwrapping process for subtracting or adding the multiple values of $2 \pi$ is needed to reconstruct the non-ambiguous phase map. The Gold-Stein phase unwrapping algorithm was employed to extract the unwrapped phase map [3]. Then, the unwrapped phase difference can be transferred to a height map using the following phase to height transformation.

The phase-to-height transformation is performed using the triangulation technique. The height information of object surface $h(x, y)$ of each pixel is carried by its phase difference value $\Delta \phi(x, y)$, which is measured by the phase difference between the measured phase on the object surface and the reference plane. Since the measured height is generally much smaller than the projection height of the optical system and the projecting objective is telecentric, the object height has a linear relation with the unwrapped phase difference as shown in Equation (3). Thus, the phase value can be transformed by a linear transformation coefficient $K$, and the height information of the object is then given as: 


$$
h(x, y)=K \Delta \phi(x, y)
$$

\subsubsection{Dual-Sensing Technique}

As mentioned above, when the projected light beam hits the tested wafer surface, the reflected light beams may contain both diffuse and specular components (see Figure 2). The diffuse components are mainly formed by reflected light beams from micro structures on wafer surface with high surface roughness. Each reflected beam may interfere with other beams. However, there is no light interference due to low light coherence. In the optical design, the diffuse light intensity is reflected at a wide reflecting angle and can be detected by a vertical sensing camera. On the other hand, specular light is generated by the reflection of incident light from an object with a shiny reflective surface on a flat wafer surface having very low surface roughness. According to the law of reflection, specular light can be detected by an optical objective located at a reflectance angle equal to that of the incident light.

To capture both diffuse and specular components simultaneously, a dual-sensing optical system is developed. In the configuration, a camera (10) is dedicated to capturing diffuse components with another camera (11) designated for capturing specular components. To reconstruct the 3-D shape of the measured surface, the reflected fringe images representing both diffuse and specular beams are simultaneously captured using the proposed system, illustrated in Figure 3. By controlling the adequate duration of light exposure required by the individual cameras, two deformed fringe images with high contrast can be detected. The entire 3-D shape of the measured target surface can be effectively synthesized from the two individual images detected and reconstructed by accurate pixel spatial mapping between two sensors. Details of the proposed measuring system are illustrated by the flowchart shown in Figure 4.

Calibrate two cameras to capture the same FOV on measured object

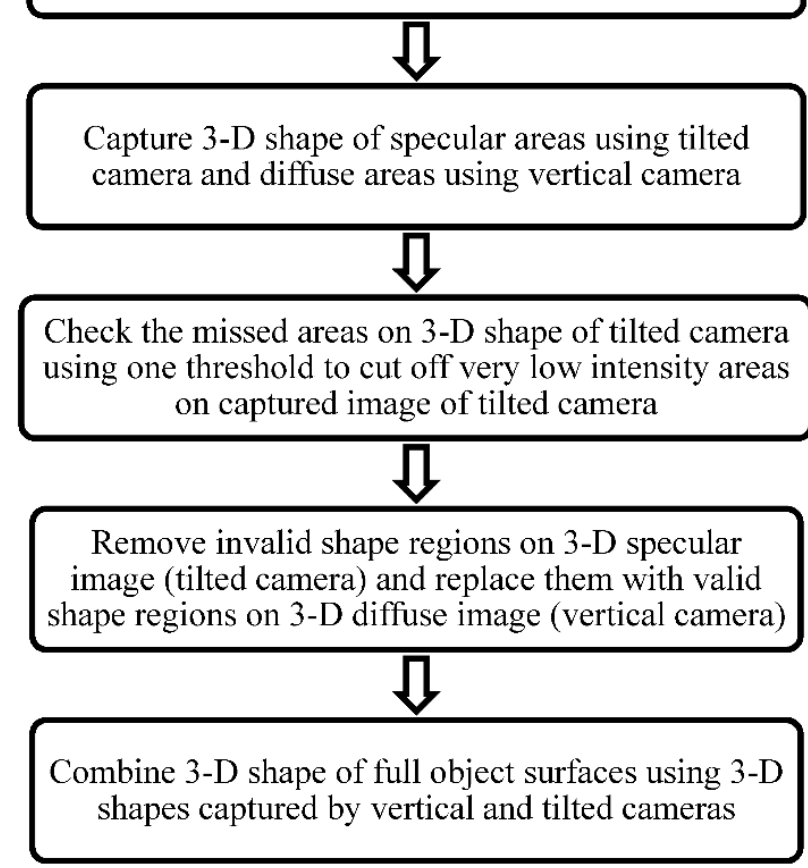

Figure 4. Flowchart of the proposed measuring technique.

In the proposed technique, the 3-D shape region detected and established from diffuse light can be well synthesized with the 3-D shape region detected and established from specular light. The two surface regions detected can be further merged seamlessly using a camera calibration procedure. 
A special artifact target is designed and employed as a special mapping target. Two cameras can be calibrated and merged into the same measuring field of view (FOV) on the tested surface. Details of the proposed calibration procedure are illustrated by the flowchart shown in Figure 5.

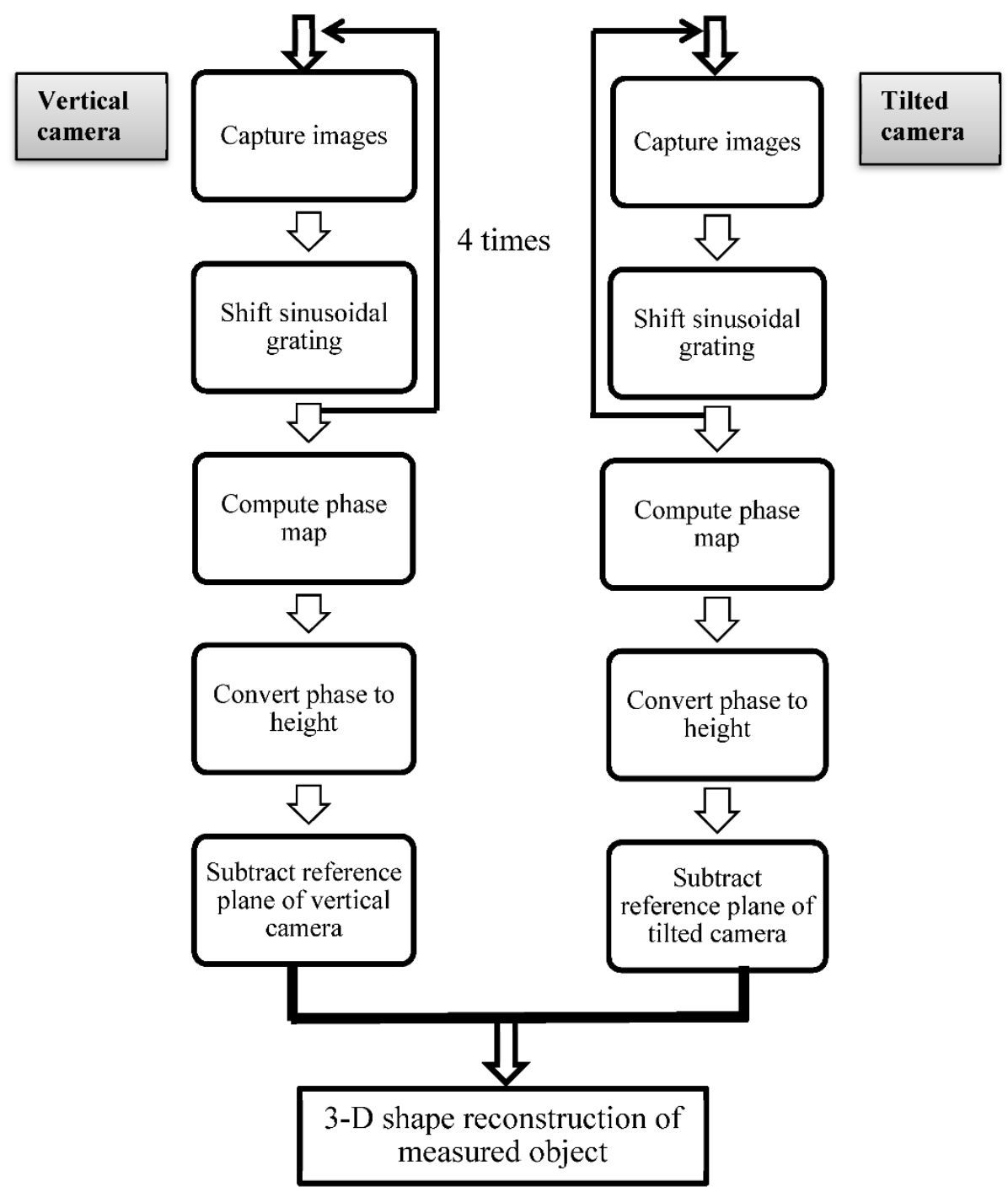

Figure 5. Flowchart of the proposed calibration procedure.

\subsubsection{Dual Camera Mapping}

To achieve precise mapping and calibration, an automatic and accurate mapping algorithm was developed to align the two cameras into the same FOV on the measured object. Figure 6 illustrates the proposed idea of dual camera mapping using random speckles. When mapping images captured by the two cameras, a unique speckle pattern is used and a cross-correlation algorithm is employed to compute the corresponding coefficient. In this study, the following normalized cross-correlation (NCC) algorithm is employed to compute the correlation between images captured by the two cameras.

$$
R(x, y)=\frac{\sum_{x^{\prime} y^{\prime}}\left(T\left(x^{\prime}, y^{\prime}\right) \cdot I^{\prime}\left(x+x^{\prime}, y+y^{\prime}\right)\right)}{\sqrt{\sum_{x^{\prime}, y^{\prime}} T\left(x^{\prime}, y^{\prime}\right)^{2} \cdot \sum_{x^{\prime}, y^{\prime}} I^{\prime}\left(x+x^{\prime}, y+y^{\prime}\right)^{2}}}
$$

where:

$$
I^{\prime}\left(x+x^{\prime}, y+y^{\prime}\right)=I\left(x+x^{\prime}, y+y^{\prime}\right)-\frac{1}{w \cdot h} \cdot \sum_{x^{\prime \prime}, y^{\prime \prime}} I^{\prime}\left(x+x^{\prime \prime}, y+y^{\prime \prime}\right)
$$




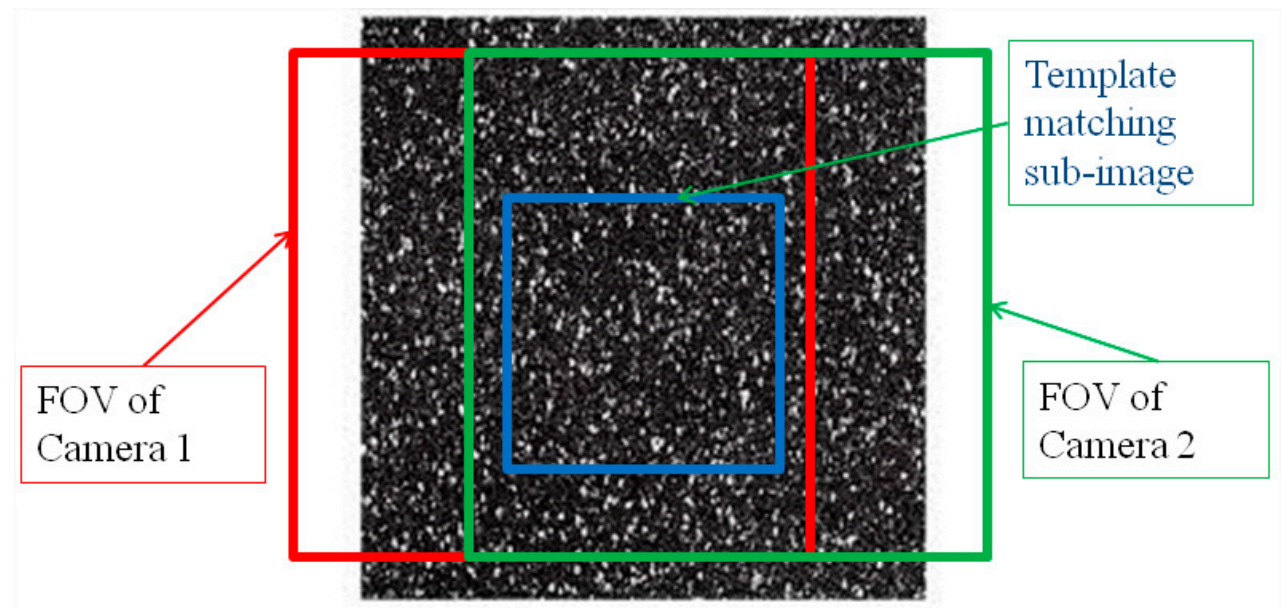

Figure 6. Proposed idea of dual camera mapping using random speckles.

The correlation value ranges from 0.0 to 1.0. The matching score of 1.0 means $100 \%$ matching between sub-image and template image. To ensure that the dual camera mapping technique worked well, a correlation threshold is needed to estimate the matching score. It is also important to note that the sub-image size is set to be large enough to retain the accuracy of the matching process. When the sub-image size is too small, the robustness of the correlation technique cannot be assured. In practice, the sub-image size is chosen as a quarter of the captured FOV to achieve good matching and reasonable computation efficiency. To achieve precise image mapping between the two images, a random speckle pattern can be prepared and employed by projecting uniform blue light on an electrostatic-coating aluminum surface with micro random patterns on it. The unique and random speckle pattern can provide accurate image registration for image mapping. Figure 7 shows the aluminum surface target designed and one of the speckle images prepared for image registration.

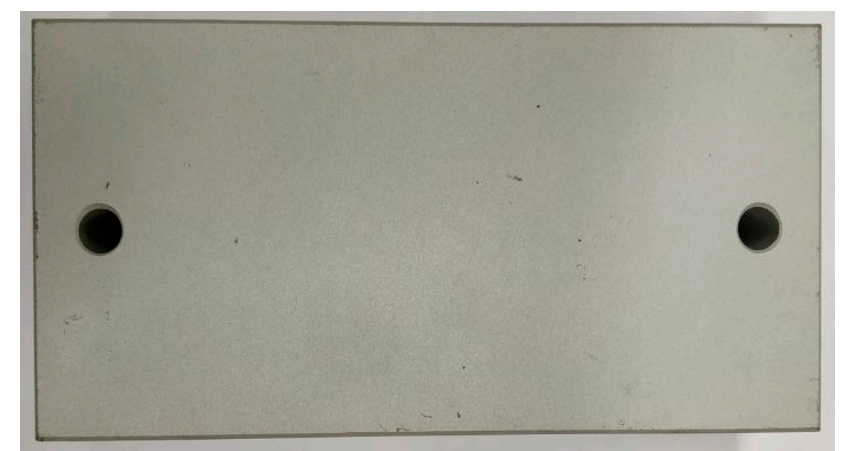

(a)

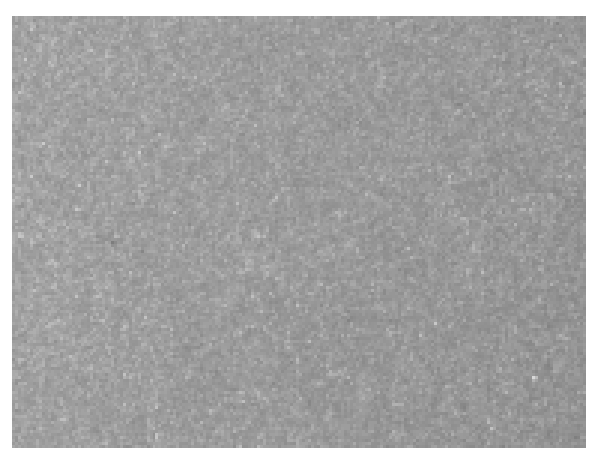

(b)

Figure 7. Calibration target design for dual camera image mapping: (a) white painted aluminum target; (b) captured speckle image using the vertical camera.

Figure 8 shows the flowchart of the proposed dual camera mapping technique. One sub-image (a quarter of captured image) of the vertical camera is cropped in the image center and selected as a template image. The matching score between the tilted image and template image (vertical camera) is determined using the NCC algorithm. The shifted value between the template image and that captured by the tilted camera can be calculated along the $\mathrm{X}$ and $\mathrm{Y}$ directions of image coordinates. This parameter can accurately represent the spatial location difference between the FOV of the tilted and the vertical cameras. This process is repeated until the parameter converged to a preset threshold for completion. 


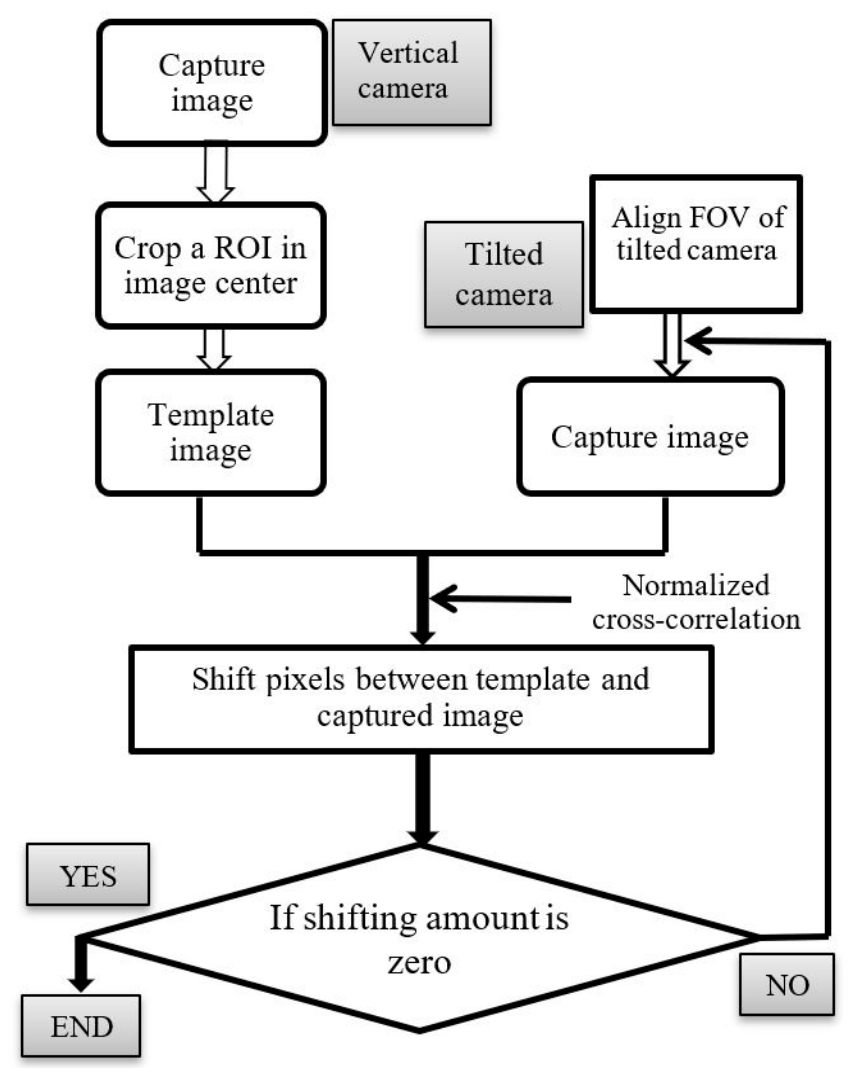

Figure 8. Flowchart of the proposed dual camera mapping procedure.

\subsubsection{Segmentation of Specular and Diffuse Images}

An image averaging algorithm is developed to extract specular and diffuse measuring regions with high $\mathrm{S} / \mathrm{N}$ ratio from the acquired deformed fringe images. Removal of the projected fringe from the acquired fringe is essential for segmentation because these deformed fringes contain light intensity modulation, thus making segmentation difficult. In phase shifting, several phase-shifted sinusoidal patterns are projected onto the tested object and their corresponding deformed fringe images are acquired sequentially. A background image is defined here as an illuminated object image without structured fringes. To obtain the background image of these deformed fringe images, the image averaging algorithm can be performed by simply summing all the phase-shifted images to remove the fringe modulation and generate the background image.

It is important to note that the above algorithm can reliably work even when the reflectivity of the tested surface is extremely variant. The algorithm operates on every individual pixel for the summing; hence, the result is generally insensitive to the pixel intensity. In an experimental test on the algorithm, the sinusoidal projection grating was made by a precise lithography process and a semiconductor's wafer surface having laser-etched groves was tested. A precise linear stage with positioning repeatability less than $1 \mu \mathrm{m}$ was adopted to perform four-step phase shifting. Figure $9 \mathrm{a}-\mathrm{d}$ illustrates the captured images from the four-step fringe projection with phase-shift $0^{\circ}, 90^{\circ}, 180^{\circ}$, and $270^{\circ}$, respectively. As can be seen, all the projected fringes are effectively removed with the image averaging algorithm, and the illustrated object image (also called the background image) is extracted. The experiment result shown in Figure 9 was acquired by the tilted camera for specular light detection on a smooth wafer surface. An excellent image contrast between specular and diffuse measured regions can be achieved in the background image. The same can be performed on the diffuse images to achieve equal effectiveness. 


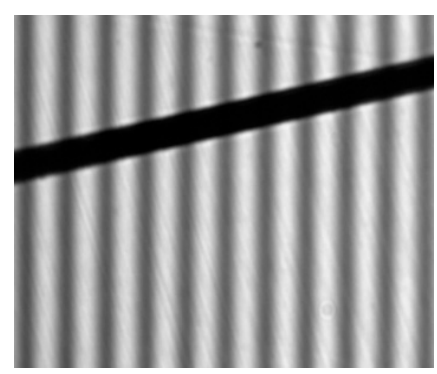

(a)

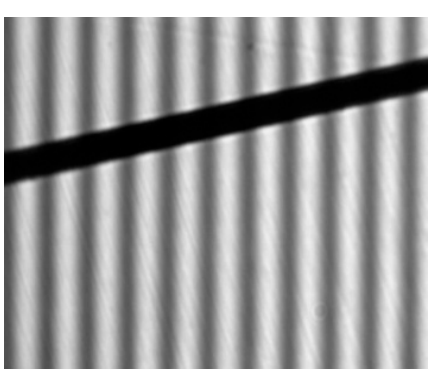

(b)

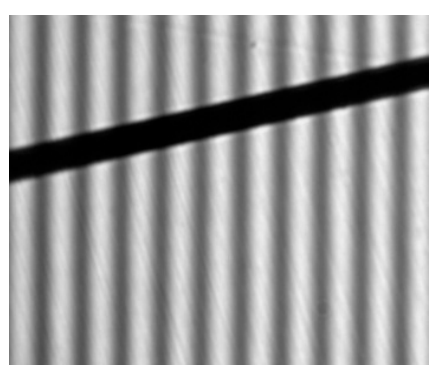

(c)

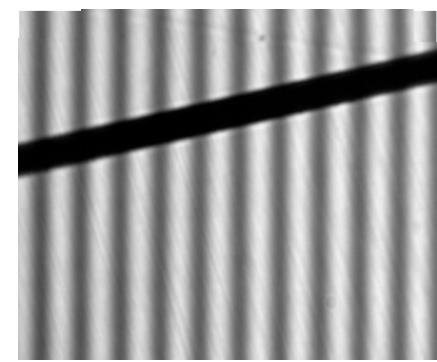

(d)

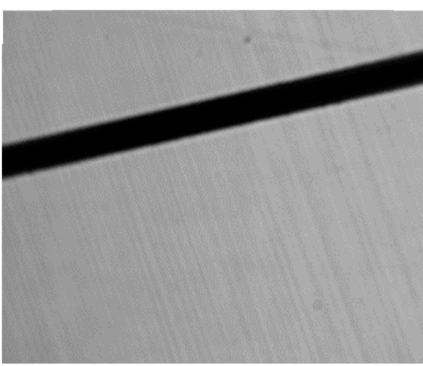

(e)

Figure 9. Phase-shifted images acquired from four-step phase shifting operation and image summing algorithm: (a) Phase-shift $0^{\circ}$; (b) Phase-shift $90^{\circ}$; (c) Phase-shift $180^{\circ}$; (d) Phase-shift $270^{\circ}$; and (e) Image result of average 4 channels.

\section{Experimental Design and Result Analyses}

To demonstrate the feasibility and measurement performance of the proposed technique, a 3-D measurement system consisting of two sensing cameras, a single-frequency transmission grating with a period of 24 line pairs per millimeter, and a step motor with positioning accuracy less than $1 \mu \mathrm{m}$ was developed. Two telecentric lenses with the same optical specification are integrated in front of the two cameras as the optical objective to collimate the reflected diffuse and specular light from the measured object surface. The light module was powered by a 30-Watt white light LED, in which the LED is mounted on a specially designed mechanical radiator for good heat dissipation. A SolidWorks design and hardware of the housing and optical system were implemented and shown in Figure 10a,b, respectively. The measuring FOV can reach $1.0 \times 1.0 \mathrm{~mm}$ for every measurement. A maximum measuring depth of the system can reach $1.0 \mathrm{~mm}$ which is sufficient for most microstructure profile inspection.

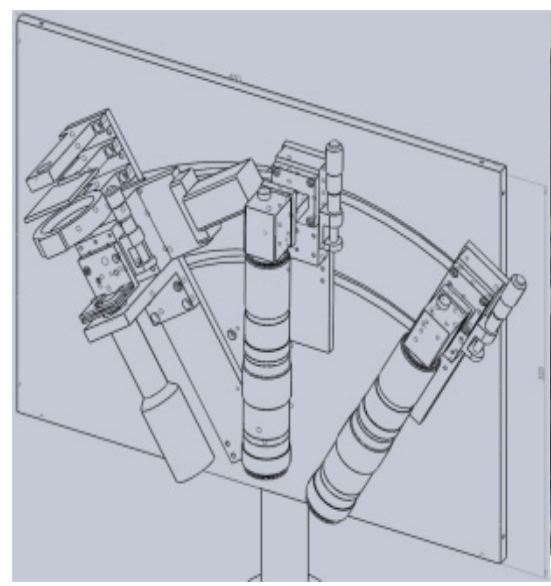

(a)

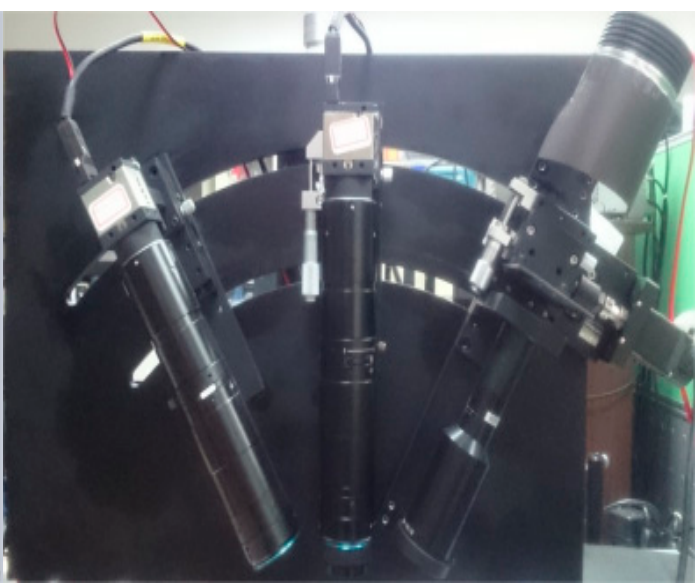

(b)

Figure 10. Developed system: (a) SolidWorks design; (b) system hardware. 
Figure 11a,b shows the fringe images of the tested wafer surface captured using the tilted and vertical cameras, respectively. Figure 11c,e illustrates the phase map and one height cross-section of the specular wafer surface measured using the tilted camera. Similarly, the phase map and one height cross-section for the laser-etched groove surface regions are measured using the vertical camera simultaneously and shown in Figure 11d,f. The 3-D profile of groove surface region can be well measured and reconstructed (see Figure 11h). It is important to note that the 3-D measured shape area of the groove region has random noises due to the non-detectable diffuse light from the laser groove surface region. Nevertheless, this region can be totally recovered and reconstructed by another vertical camera which can capture the diffuse light from the groove region. Two detected 3-D profiles, shown in Figure 11g,h, are extracted from their respective original images by the image summing algorithm and further mapped by the camera calibration technique precisely to reconstruct a full-field 3-D profile of the measured wafer, shown in Figure 11i. These results indicate that the full-field 3-D wafer surface can be measured and reconstructed accurately using the developed technique.

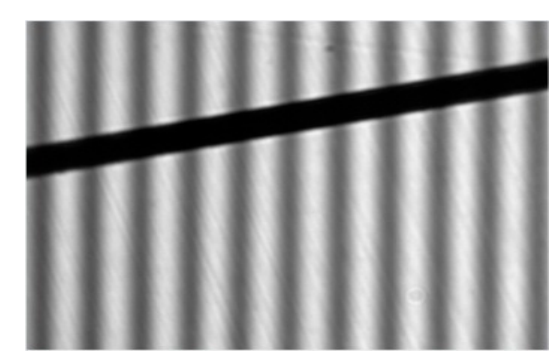

(a)

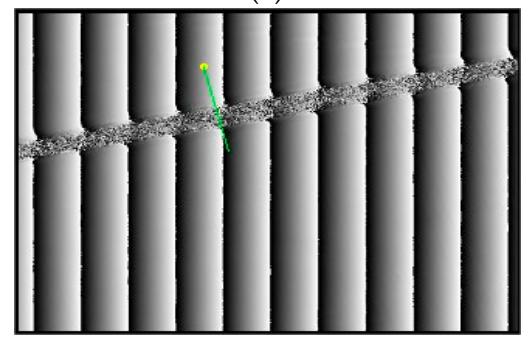

(c)

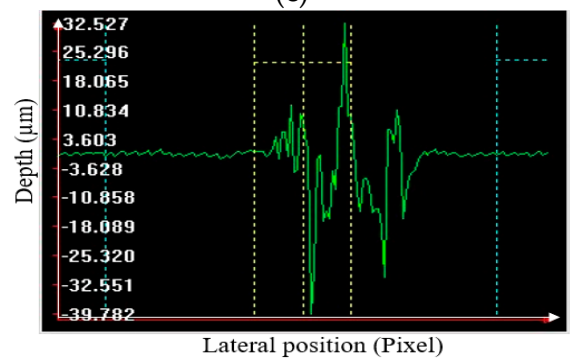

(e)

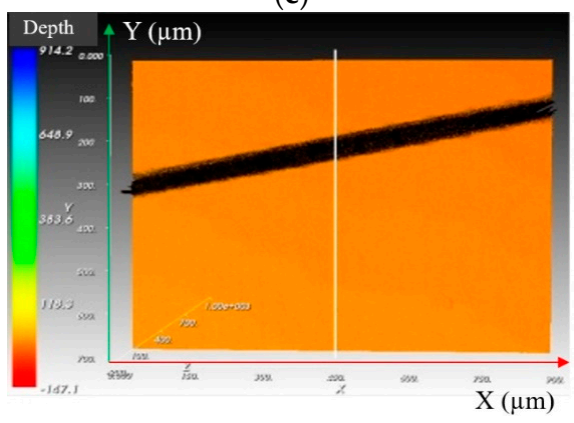

(g)

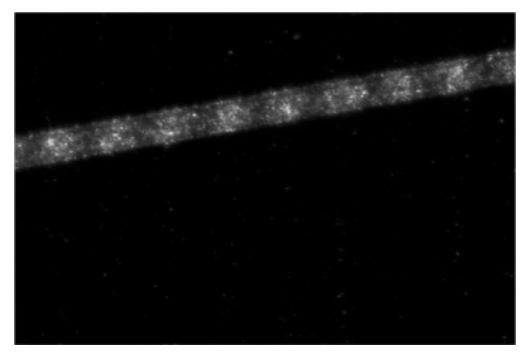

(b)

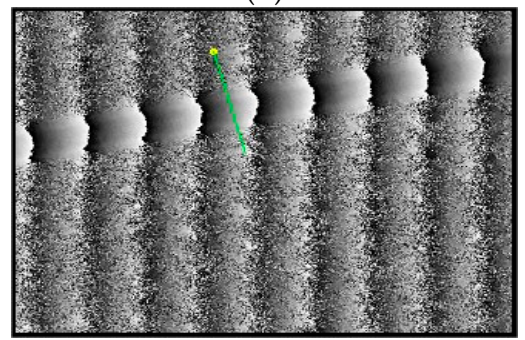

(d)

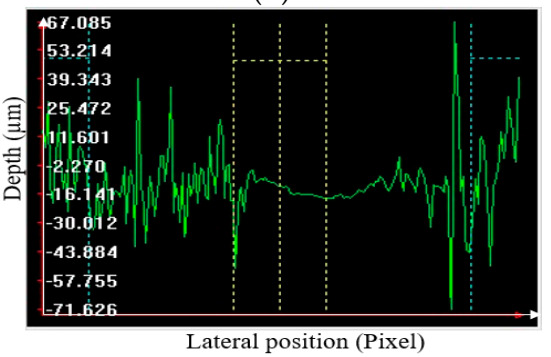

(f)

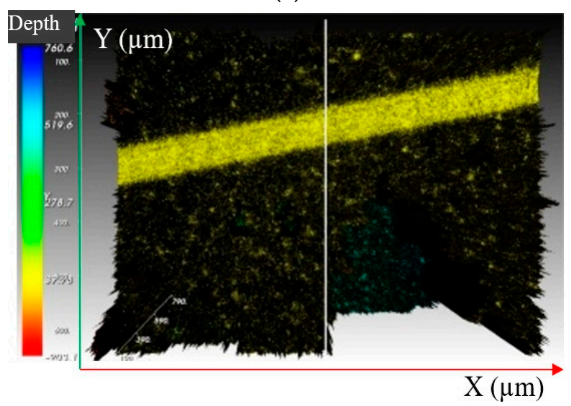

(h)

Figure 11. Cont. 


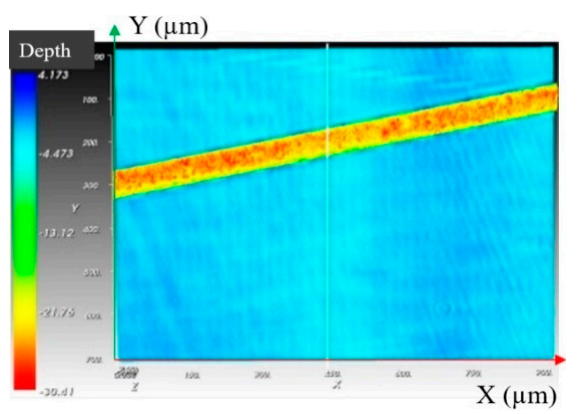

(i)

Figure 11. Measurement results of the tested wafer sample shown in Figure 1: (a) captured deformed fringe image of specular light, (b) captured deformed fringe image of diffuse light, (c) wrapped phase map of $(\mathbf{b}, \mathbf{d})$ wrapped phase map of $(\mathbf{c}, \mathbf{e})$ reconstructed cross-section of $(\mathbf{c}, \mathbf{f})$ reconstructed cross-section of $(\mathbf{d}, \mathbf{g})$ reconstructed 3-D map of the smooth wafer surface, (h) reconstructed 3-D map of the laser-etched groove surface, and (i) reconstructed 3-D shape of the whole tested wafer surface.

Figure 12 exhibits the measured height cross-section along one lateral section on the tested wafer being captured by the tilted and vertical cameras. The standard deviation of the wafer surface region and groove area are evaluated for measurement precision. In a 30-time repeatability test, the standard deviation in the measured data region, shown in Figure 12a,b, are $0.265 \mu \mathrm{m}$ and $1.937 \mu \mathrm{m}$, respectively.

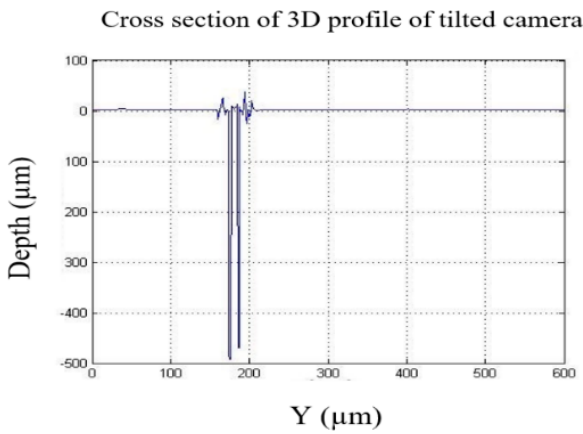

(a)

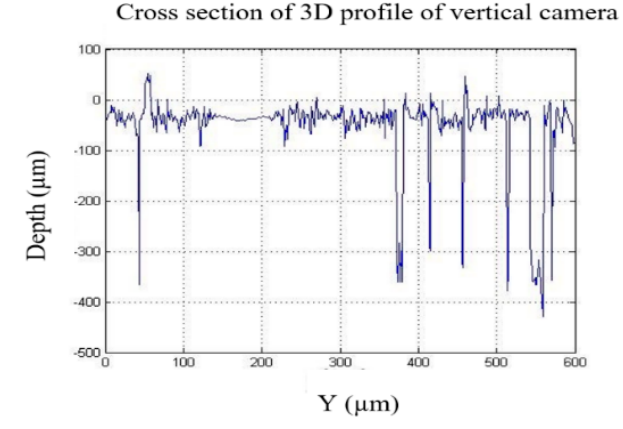

(b)

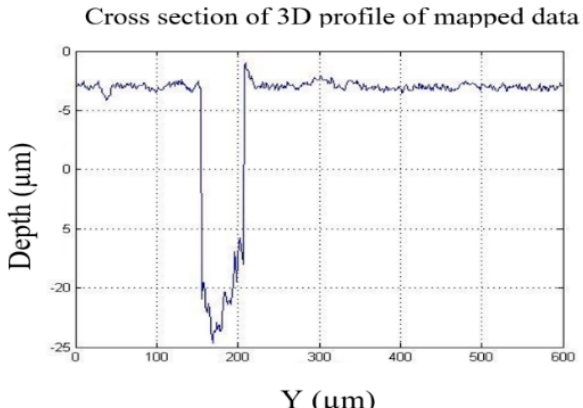

(c)

Figure 12. Height cross-section of a surface cross-section on the wafer in Figure 1: (a) reconstructed cross-section profile by capturing specular light. (b) reconstructed cross-section profile by capturing diffuse light, and (c) mapped cross-section profile.

The results obtained show that the measurement repeatability can be achieved for less than $2.0 \mu \mathrm{m}$ when measuring a rough laser-machined wafer surface. With the proposed technique, the measured average step height of the cutting groove on the wafer is $19.01 \mu \mathrm{m}$.

To check the measurement accuracy, the measured result was compared with a pre-calibrated laser confocal microscope. Figure 13 illustrates the measurement result obtained using a laser confocal 
microscope (KEYENCE VK-X1000) on the same measured wafer surface region. The measured average step height of the laser-etched groove in a 30-time measurement was $18.853 \mu \mathrm{m}$. With the developed method, the measured average step height on the same wafer surface was $20.21 \mu \mathrm{m}$, in which the measurement deviation in this case was $1.357 \mu \mathrm{m}$.

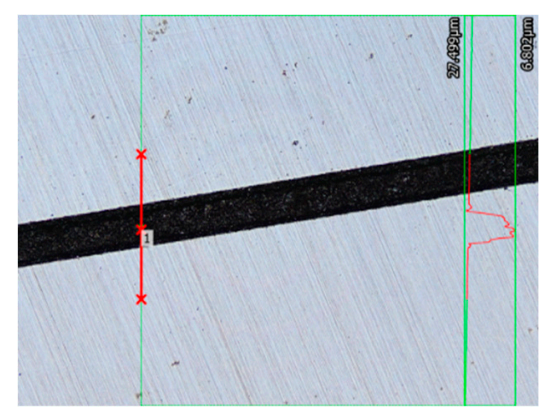

(a)

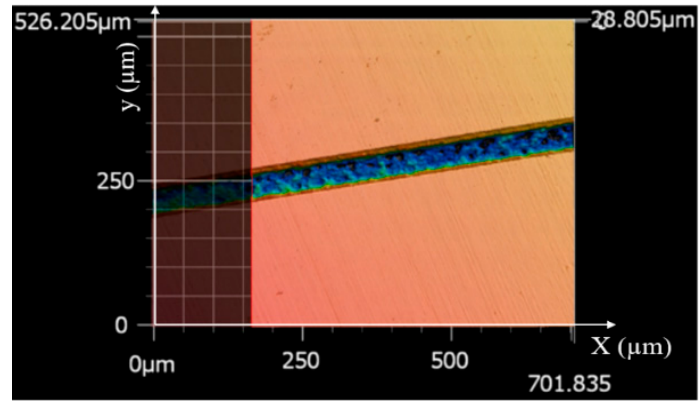

(b)

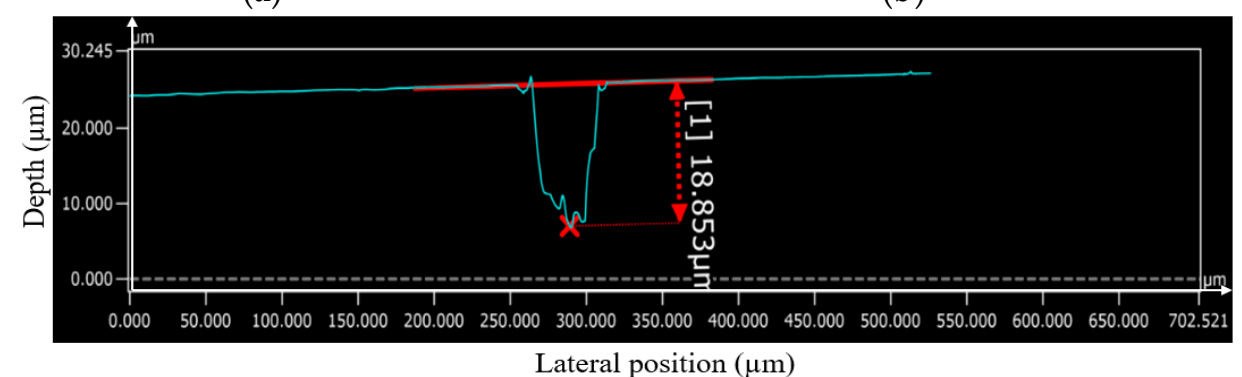

(c)

Figure 13. Measurement results of the wafer obtained using a laser confocal microscope (KEYENCE VK-X1000): (a) 2D captured image of the wafer, (b) 3-D measured profile of wafer, and (c) one cross-section of the 3-D profile.

To further check the feasibility of the developed method on an industrial semiconductor parts with variant surface reflectivity, an IC chip was selected for measuring its surface profile. Figure 14 shows the measured results on an industrial IC surface with extreme reflectivity variance between the substrate and the metal pads. The substrate image detected by specular light is shown in Figure 14a while the diffuse image detected by diffuse light is illustrated in Figure 14b. Neither of these two images can cover the entire surface detection. With the developed method, both specular and diffuse images can be detected simultaneously and reconstructed accurately. The phase map and one surface cross-section profile can be reconstructed for smooth substrate surface regions, as seen in Figure 14c,e, respectively. Similarly, Figure $14 \mathrm{~d}$, f represent respectively the phase map and one surface cross-section profile of the metal-pad regions. Compared with the developed method, the traditional fringe projection method will find excessive noises measured around the IC chip's pads which are mainly reflected diffuse light areas, as seen in Figure 14g. By using the developed technique, the surface measurement of the IC chip can be successfully obtained, in which both the pad and substrate areas can be reconstructed simultaneously and accurately, as shown in Figure 14j. In other words, a significant improvement in surface profilometry of objects with substantial variance of surface reflectance can be achieved with the developed approach. 


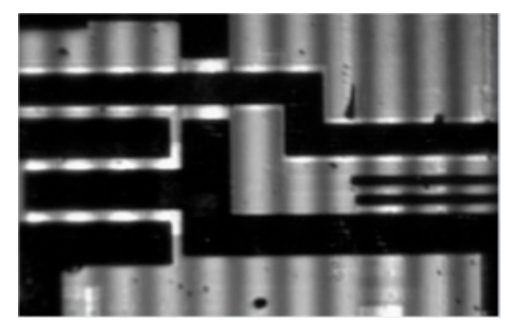

(a)

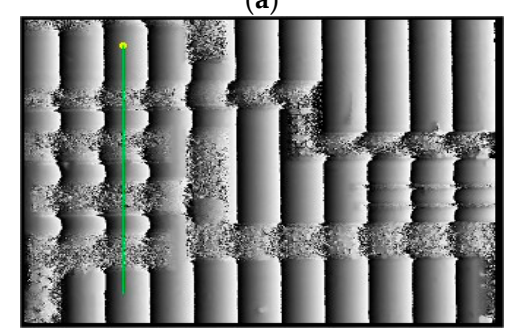

(c)

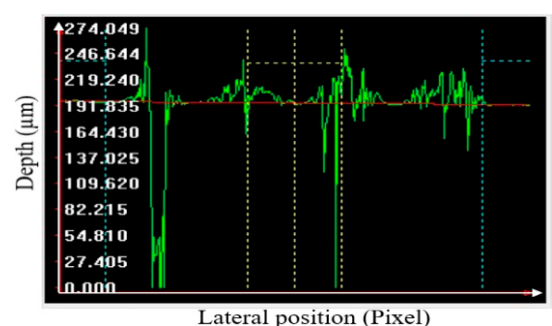

(e)

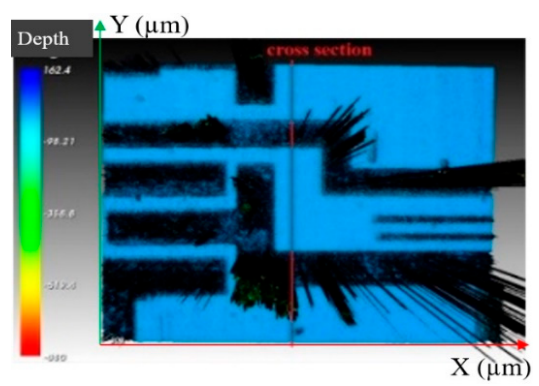

(g)

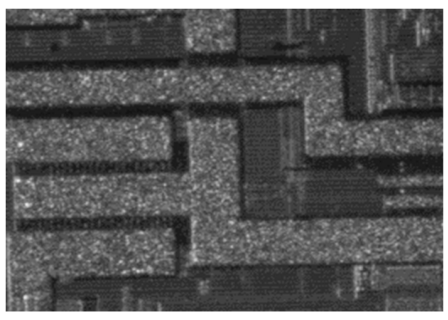

(i)

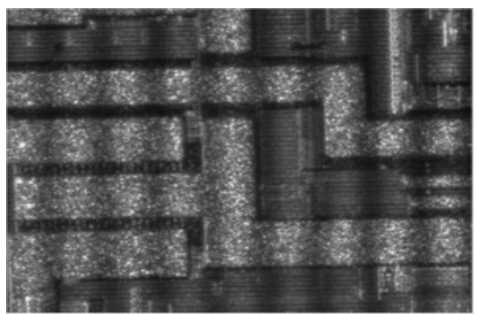

(b)

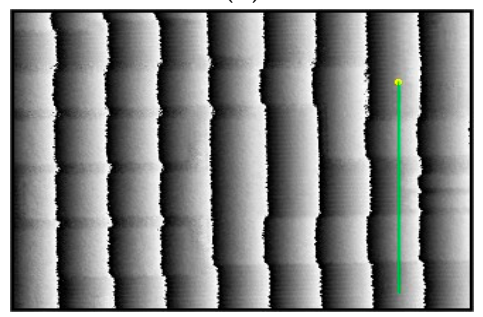

(d)

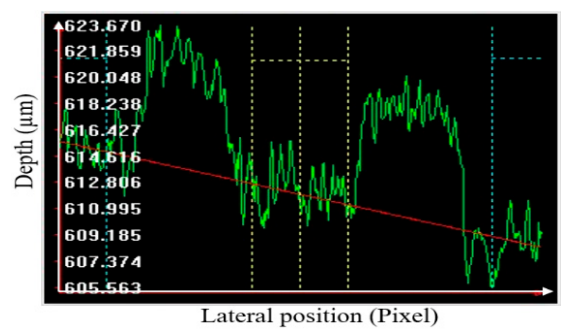

(f)

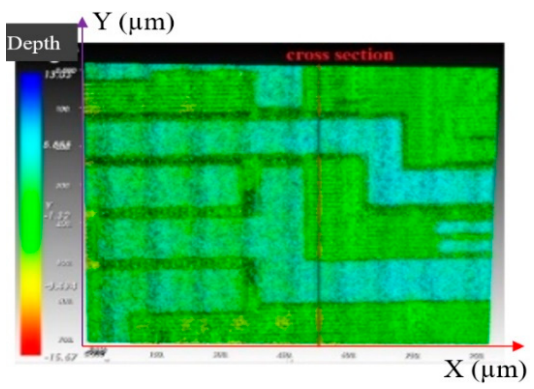

(h)

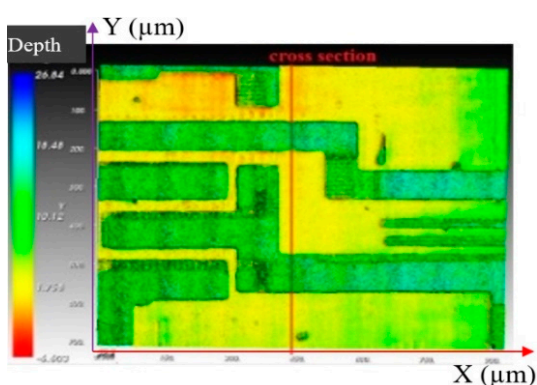

(j)

Figure 14. Measurement results of industrial IC chip: (a) image captured by camera receiving specular light; (b) image captured by vertical camera; (c) wrapped phase map of tilted camera; (d) wrapped phase map of vertical camera; (e) cross-section on wrapped phase map of tilted camera; (f) cross-section on wrapped phase map of vertical camera; (g) 3-D shape of IC chip measured by tilted camera; (h) 3-D shape of IC chip measured by vertical camera; (i) measured ROI of industrial IC chip; and, (j) 3-D shape of measured ROI in (i). 
Figure 15a,b illustrate the cross-section of height map at Column 400 on the IC chip imaged by the two cameras in charge of capturing specular and diffuse light, respectively. With the mapping algorithm, the merged cross-section profile can be obtained, as seen in Figure 15c. The measurement standard deviations of the specular and diffuse surfaces on the IC chip are 0.602 and $1.285 \mu \mathrm{m}$, respectively. With the proposed technique, the measured average step height of the metal pads on the IC chip is $13.2 \mu \mathrm{m}$.

Cross section of 3D profile of tilted camera

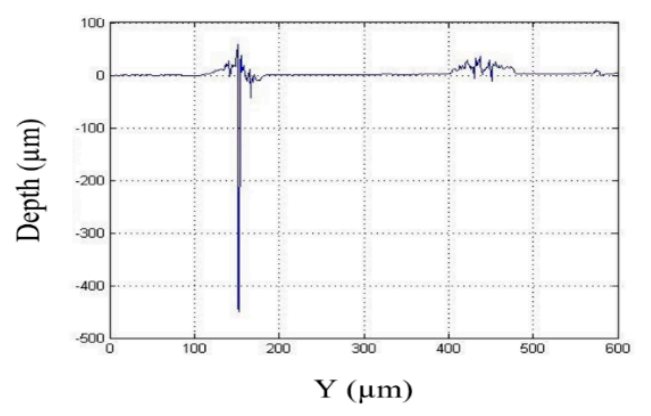

(a)

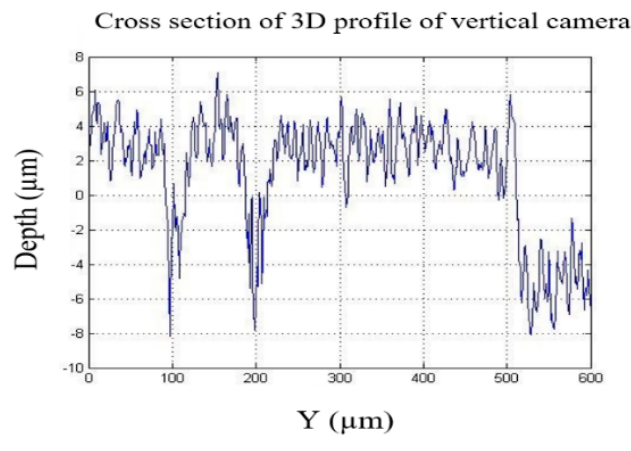

(b)

Cross section of 3D profile of mapped data

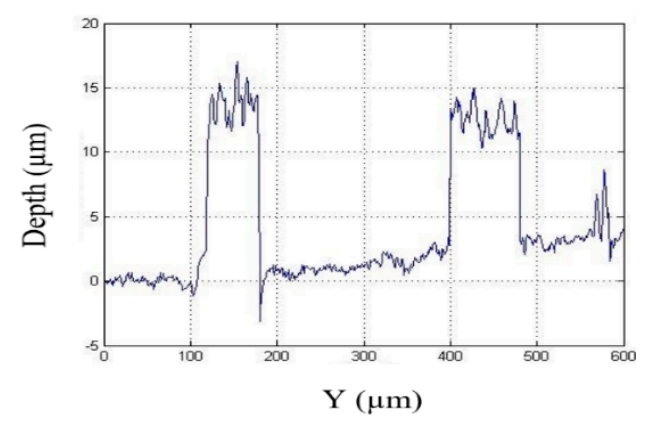

(c)

Figure 15. Cross-section profile measured on IC chip shown in Figure 14: (a) cross-section profile reconstructed from specular light components; (b) cross-section profile reconstructed from diffuse light components; (c) 3-D cross-section surface mapped by proposed mapping algorithm.

\section{Conclusions}

In this study, a new dual-sensing measuring technique for 3-D surface profilometry is developed and proved effective for measuring objects with extreme surface reflectance variance. The key element of the developed technique is its new optical configuration for simultaneous detection of both diffuse and specular light reflected from various surface regions on a tested object. Moreover, an accurate image segmentation and mapping algorithm is developed to extract effectively detected image regions and merge them seamlessly into an accurate 3-D surface map. Experimental results indicate that the proposed technique can successfully achieve simultaneous measurement with repeatability of one standard deviation below $0.3 \mu \mathrm{m}$ on a specular surface and $2.0 \mu \mathrm{m}$ in a total vertical measurable range of $1.0 \mathrm{~mm}$ on a diffuse surface. Specular light detection is more accurate than diffuse light detection in 3-D surface profilometry, which is mainly influenced by the SNR (signal-to-noise ratio) of the deformed fringe image. The developed technique can provide an effective wafer surface measuring technique for in-situ AOI, especially in 3-D full-field surface profilometry.

Author Contributions: Conceptualization, L.-C.C.; Methodology, L.-C.C. and D.-H.D. ; Software, L.-C.C. and D.-H.D.; Validation, L.-C.C. and D.-H.D.; Formal Analysis, L.-C.C. and D.-H.D.; Investigation, L.-C.C., D.-H.D. and C.-S.C.; Resources, L.-C.C.; Data Curation, L.-C.C. and D.-H.D.; Writing-Original Draft Preparation, L.-C.C. 
and D.-H.D.; Writing-Review \& Editing, L.-C.C.; Visualization, L.-C.C.; Supervision, L.-C.C. and C.-S.C.; Project Administration, L.-C.C. and C.-S.C.; Funding Acquisition, L.-C.C.

Funding: This research was funded by Ministry of Science and Technology, Taiwan, under grant no. MOST 107-2218-E-002-060 and MOST 107-2218-E-002-002.

Conflicts of Interest: The authors declare no conflict of interest.

\section{References}

1. Gåsvik, K.J. Optical Metrology, 3rd ed.; John Wiley \& Sons Ltd., Ed.; Wiley Online Library: Chichester, UK, 2003; pp. 173-190.

2. Takeda, M.; Mutoh, K. Fourier transform profilometry for the automatic measurement of 3-D object shapes. Appl. Opt. 1983, 22, 3977-3982. [CrossRef] [PubMed]

3. Ghiglia, D.C.; Pritt, M.D. Two-Dimensional Phase Unwrapping: Theory, Algorithms, and Software; John Wiley \& Sons Ltd., Ed.; Wiley Online Library: New York, NY, USA, 1998; ISBN 978-0-471-24935-1.

4. Chen, L.-C.; Nguyen, X.-L.; Ho, H.-W. High-speed 3-D machine vision employing Fourier Transform Profilometry with digital tilting-fringe projection. In Proceedings of the 2008 IEEE Workshop on Advanced robotics and Its Social Impacts, Taipei, Taiwan, 23-25 August 2008; pp. 1-6.

5. Gdeisat, M.A.; Abid, A.; Burton, D.R.; Lalor, M.J.; Lilley, F.; Moore, C.; Qudeisat, M. Spatial and temporal carrier fringe pattern demodulation using the one-dimensional continuous wavelet transform: Recent progress, challenges, and suggested developments. Opt. Lasers Eng. 2009, 47, 1348-1361. [CrossRef]

6. Gorecki, C. Interferogram analysis using a Fourier transform method for automatic 3D surface measurement. Pure Appl. Opt. J. Eur. Opt. Soc. Part A 1992, 1, 103. [CrossRef] [PubMed]

7. Lin, J.-F.; Su, X. Two-dimensional Fourier transform profilometry for the automatic measurement of three-dimensional object shapes. Opt. Eng. 1995, 34, 34-36.

8. Chen, L.-C.; Ho, H.-W.; Nguyen, X.-L. Fourier transform profilometry (FTP) using an innovative band-pass filter for accurate 3-D surface reconstruction. Opt. Lasers Eng. 2010, 48, 182-190. [CrossRef]

9. Chen, L.-C.; Hai, H.H. Fourier transform profilometry employing novel orthogonal elliptic band-pass filtering for accurate 3-D surface reconstruction. Precis. Eng. 2014, 38, 512-524. [CrossRef]

10. Chen, L.-C.; Ho, H.-W. Method for Acquiring Phase Information and System for Measuring Three Dimensional Surface Profiles. Patent US8385629 B2, 26 February 2013.

11. Wyant, J.C. White light interferometry. Proc. SPIE 2002, 4737, 98-107.

12. Artusi, A.; Banterle, F.; Chetverikov, D. A survey of specularity removal methods. In Computer Graphics Forum; Wiley Online Library: New York, NY, USA, 2011; Volume 30, pp. 2208-2230.

13. Kim, D.W.; Lin, S.; Hong, K.-S.; Shum, H.Y. Variational specular separation using color and polarization. In Proceedings of the IAPR Workshop on Machine Vision Applications, Nara, Japan, 11-13 December 2002; pp. 176-179.

14. Umeyama, S.; Godin, G. Separation of diffuse and specular components of surface reflection by use of polarization and statistical analysis of images. IEEE Trans. Pattern Anal. Mach. Intell. 2004, 26, 639-647. [CrossRef] [PubMed]

15. Ma, W.-C.; Hawkins, T.; Peers, P.; Chabert, C.-F.; Weiss, M.; Debevec, P. Rapid Acquisition of Specular and Diffuse Normal Maps from Polarized Spherical Gradient Illumination. In Proceedings of the 18th Eurographics Conference on Rendering Techniques, Grenoble, France, 25-27 June 2007; Eurographics Association: Aire-la-Ville, Switzerland, 2007; pp. 183-194.

16. Tan, R.T.; Ikeuchi, K. Separating reflection components of textured surfaces using a single image. IEEE Trans. Pattern Anal. Mach. Intell. 2005, 27, 178-193. [CrossRef] [PubMed]

17. Zhang, S.; Yau, S.-T. High dynamic range scanning technique. In Proceedings of the Mechanical Engineering Conference Presentations, San Diego, CA, USA, 27-31 July 2008.

18. Song, Z.; Chung, R.; Zhang, X. An Accurate and Robust Strip-Edge-Based Structured Light Means for Shiny Surface Micromeasurement in 3-D. IEEE Trans. Ind. Electron. 2013, 60, 1023-1032. [CrossRef]

19. Knauer, M.C.; Kaminski, J.; Hausler, G. Phase measuring deflectometry: A new approach to measure specular free-form surfaces. Proc. SPIE 2004, 5457, 5411-5457.

20. Bothe, T.; Li, W.; von Kopylow, C.; Juptner, W.P.O. High-resolution 3D shape measurement on specular surfaces by fringe reflection. Proc. SPIE 2004, 5457, 5412-5457. 
21. Nguyen, M.T.; Ghim, Y.-S.; Rhee,H.-G. Single-shot deflectometry for dynamic 3D surface profile measurement by modified spatial-carrier frequency phase-shifting method. Sci. Rep. 2019, 9, 3157. [CrossRef] [PubMed]

22. Wiedenmann, E.; Scholz, T.; Schott, R.; Tusch, J.; Wolf, A. First Utilization of Energy Transfer in Structured Light Projection-Infrared 3D Scanner. Key Eng. Mater. 2014, 613, 141-150. [CrossRef]

23. Ihrke, I.; Kutulakos, K.; Lensch, H.P.A.; Magnor, W.H. State of the Art in Transparent and Specular Object Reconstruction. In Proceedings of the STAR Proc. of Eurographics, Crete, Greece, 14-18 April 2008.

24. Wolff, L.B. Using polarization to separate reflection components. In Proceedings of the Proceedings CVPR '89: IEEE Computer Society Conference on Computer Vision and Pattern Recognition, San Diego, CA, USA, 4-8 June 1989; pp. 363-369.

25. Lamond, B.; Peers, P.; Ghosh, A.; Debevec, P.D. Image-based Separation of Diffuse and Specular Reflections using Environmental Structured Illumination. In Proceedings of the 2009 IEEE International Conference on Computational Photography (ICCP), San Francisco, CA, USA, 16-17 April 2009; IEEE: San Francisco, CA, USA, 2009.

26. SCILLC. Soldering and Mounting Techniques. Reference manual; Rev. 11, SOLDERRM/D; On semiconductor Ltd.: Phoenix, AZ, USA, March 2016; pp. 1-82.

(C) 2019 by the authors. Licensee MDPI, Basel, Switzerland. This article is an open access article distributed under the terms and conditions of the Creative Commons Attribution (CC BY) license (http://creativecommons.org/licenses/by/4.0/). 